\title{
GREEN'S FUNCTION IN FREQUENCY ANALYSIS OF CIRCULAR THIN PLATES OF VARIABLE THICKNESS
}

\author{
KRZYSZTOF KAMIL ŻUR \\ Bialystok University of Technology, Faculty of Management, Kleosin, Poland \\ e-mail:k.zur@pb.edu.pl
}

\begin{abstract}
Free vibration analysis of homogeneous and isotropic circular thin plates with variable distribution of parameters by using Green's functions (solution to homogeneous ordinary differential equations with variable coefficients) is considered. The formula of Green's function (called the influence function) depends on the Poisson ratio and the coefficient of distribution of plate flexural rigidity, and the thickness is obtained in a closed-form. The limited independent solutions to differential Euler equations are expanded in the Neumann power series using the Volterra integral equations of the second kind. This approach allows one to obtain the analytical frequency equations as the power series rapidly convergens to exact eigenvalues for different values of the power index and different values of the Poisson ratio. The six lower natural dimensionless frequencies of axisymmetric vibration of circular plates of constant and variable thickness are calculated for different boundary conditions. The obtained results are compared with selected results presented in the literature.
\end{abstract}

Keywords: circular plates, Green's function, Neumann series

\section{Introduction}

The study of vibration of a thin circular plate is basic in structural mechanics because it has many applications in civil and mechanical engineering. Circular plates are the most critical structural elements in high speed rotating engineering systems such as circular saws, rotors, turbine flywheels, etc. In reality, a lot of complicating factors may come into play: non-uniform thickness, elastic constraints, anisotropic or composite materials, etc. The natural frequencies of the plates have been studied extensively for more than a century, if only because when the frequency of external load matches the natural frequency of the plate, destruction may occur.

The free vibration of circular plates of constant and variable thickness has received considerable attention in the literature. The vibration of circular plates has been discussed by many authors. The work of Leissa (1969) is an excellent source of information about methods used for free vibration analysis of plates. Free vibration analysis has been carried out by using a variety of weighting function methods (Leissa, 1969) such as the Ritz method, the Galerkin method or the finite element method. Conway $(1957,1958)$ analyzed the axisymmetric vibration of thin circular plates with a power function thickness variation for a particular Poisson ratio in terms of the Bessel functions. Jain et al. (1972) studied the axisymmetric vibration of thin circular plates with linearly varying thickness using by the Frobenius method. Yang (1993) studied the same problem using by perturbation method. Wang (1997) used the power series method for free vibration analysis of circular thin plates with power variable thickness. Wu and Liu (2001, 2002) proposed a generalized differential quadrature rule (GDQR) for free vibration analysis of circular thin plates of constant and variable thickness. Jaroszewicz and Zoryj (2006) studied free vibration of circular thin plates with variable distribution of parameters using the method of partial discretization (MPD). Taher et al. (2006) studied free vibration of circular and annular plates with variable thickness and different combinations of boundary conditions. Gupta et 
al. (2006) analyzed free vibration of nonhomogeneous circular plates with nonlinear thickness variation by using the differential quadrature method (DQM). Yalcin et al. (2009) studied free vibration of circular plates by using the differential transformation method (DTM). Zhou et al. (2011) applied the Hamiltonian approach to solution of the free vibration problem of circular and annular thin plates. Duan et al. (2014) proposed the DSC element method for free vibration analysis of circular thin plates with constant and stepped thickness.

In the works by Leissa (1969), Conway $(1957,1958)$ the solutions for free axisymmetric vibration of clamped circular plates with a power function thickness variation were presented. Those solutions were possible to obtain only for few combinations of the Poisson ratios and Bessel functions. That kind of solutions have limited practical applications. The aim of the paper is frequency analysis of circular plates with different values of the power index $m$ of the plate parameters and different values of the Poisson ratios. The characteristic equations are obtained for two different values of the Poisson ratio and different boundary conditions such as free, clamped, simply supported, sliding and elastic supports. The limited independent solutions of differential Euler equations are expanded in the Neumann power series using the properties of integral equations. This approach allows one to obtain analytical frequency equations as the power series rapidly converges to the exact eigenvalues. The numerical results of investigation are in good agreement with selected results presented in the literature.

\section{Statement of the problem}

Consider an isotropic, homogeneous circular thin plate of variable thickness $h=h_{R} r^{m / 3}$ and flexural rigidity $D=D_{R} r^{m}$ in the cylindrical coordinate system $(r, \theta, z)$ with the $z$-axis along the longitudinal direction. $h_{R}$ and $D_{R}$ are thickness and flexural rigidity of circular plates on the edge $(r=R)$, respectively. The geometry and coordinate system of the considered plate are shown in Fig. 1. For free axisymmetric vibration of circular plates, the deflection is independent of $\theta$. The partial differential equation for free vibration of thin circular plates has the following form (Timoshenko and Woinowsky-Krieger, 1959)

$$
D \frac{\partial}{\partial r}\left(\frac{\partial^{2} W}{\partial r^{2}}+\frac{1}{r} \frac{\partial W}{\partial r}\right)+\frac{\partial D}{\partial r}\left(\frac{\partial^{2} W}{\partial r^{2}}+\frac{\nu}{r} \frac{\partial W}{\partial r}\right)+\frac{1}{r} \int_{0}^{r} \rho h \frac{\partial^{2} W}{\partial t^{2}} r d r=0
$$

where $\rho$ is mass density, $r$ is the radial coordinate and $W(r, t)$ is the small axisymmetric deflection compared with the thickness $h$ of the plate.

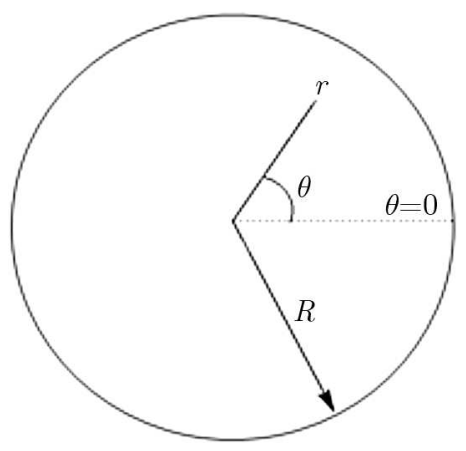

Fig. 1. Geometry and coordinate system of the circular plate

The axisymmetric deflection of a circular plate may be expressed as follows

$$
W(r, t)=w(r) \mathrm{e}^{\mathrm{i} \omega t}
$$


where $w(r)$ is the radial mode function and $\omega$ is natural frequency. Substituting Eq. (2.2) into Eq. (2.1) and using the dimensionless coordinate $\xi=r / R$, the governing differential equation of the circular plate becomes

$$
L(w)-\lambda^{2} \xi^{-2 m / 3} w=0
$$

where $L(w)$ is the operator defined by

$$
L(w) \equiv \frac{d^{4} w}{d \xi^{4}}+\frac{2(m+1)}{\xi} \frac{d^{3} w}{d \xi^{3}}+\frac{m^{2}+m+\nu m-1}{\xi^{2}} \frac{d^{2} w}{d \xi^{2}}+\frac{m^{2} \nu-m \nu-m+1}{\xi^{3}} \frac{d w}{d \xi}
$$

and the dimensionless frequency $\lambda$ of vibration is given by

$$
\lambda=\omega R^{m / 3} \sqrt{\frac{\rho h_{R}}{D_{R}}}
$$

The governing differential equation for the circular plate of constant thickness has the form

$$
L(w)-\lambda^{2} w=0
$$

where

$$
L(w) \equiv \frac{d^{4} w}{d \xi^{4}}+\frac{2}{\xi} \frac{d^{3} w}{d \xi^{3}}-\frac{1}{\xi^{2}} \frac{d^{2} w}{d \xi^{2}}+\frac{1}{\xi^{3}} \frac{d w}{d \xi} \quad \lambda=\omega R^{2} \sqrt{\frac{\rho h_{R}}{D_{R}}}
$$

The boundary conditions at the outer edge $(\xi=1)$ of the circular plate may be one of the following: clamped, simply supported, free, sliding supports and elastic supports. These conditions may be written in terms of the radial mode function $w(\xi)$ in the following form:

- clamped

$$
\left.w(\xi)\right|_{\xi=1}=\left.0 \quad \frac{d w}{d \xi}\right|_{\xi=1}=0
$$

— simply supported

$$
\left.w(\xi)\right|_{\xi=1}=\left.0 \quad M(w)\right|_{\xi=1}=\left(\frac{d^{2} w}{d \xi^{2}}+\frac{\nu}{\xi} \frac{d w}{d \xi}\right)_{\xi=1}=0
$$

- free

$$
\left.M(w)\right|_{\xi=1}=\left.0 \quad V(w)\right|_{\xi=1}=\left(\frac{d^{3} w}{d \xi^{3}}+\frac{1}{\xi} \frac{d^{2} w}{d \xi^{2}}-\frac{1}{\xi^{2}} \frac{d w}{d \xi}\right)_{\xi=1}=0
$$

— sliding (vertical) supports

$$
\left.\frac{d w}{d \xi}\right|_{\xi=1}=\left.0 \quad V(w)\right|_{\xi=1}=0
$$

— elastic supports

$$
\begin{aligned}
& \left.\Phi(w)\right|_{\xi=1}=\left[\left(\frac{d^{2} w}{d \xi^{2}}+\nu \frac{d w}{d \xi}\right)+\phi \frac{d w}{d \xi}\right]_{\xi=1}=0 \\
& \left.\Psi(w)\right|_{\xi=1}=\left[\left(\frac{d^{3} w}{d \xi^{3}}+\frac{d^{2} w}{d \xi^{2}}-\frac{d w}{d \xi}\right)-\psi w\right]_{\xi=1}=0
\end{aligned}
$$

$M(w)$ and $V(w)$ are the normalized radial bending moment and the normalized effective shear force, respectively. $\phi=K_{\phi} R / D_{R}$ and $\psi=K_{\psi} R^{3} / D_{R}$ are parameters of the elastic supports. $K_{\phi}$ and $K_{\psi}$ are the rotational and translational spring constants (Fig. 2), respectively. 


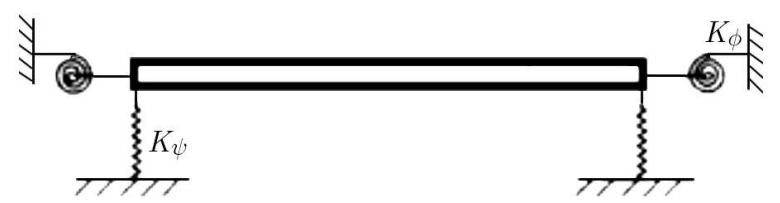

Fig. 2. Cross-section of a uniform circular plate with elastic supports

\section{Finding Green's functions}

The characteristic equation of a homogeneous differential Euler equation for thin circular plates with variable thickness, see Eq. (2.4)

$$
L(w)=0
$$

has the following form

$$
s^{4}+(2 m-4) s^{3}+\left(m^{2}+m \nu-5 m+4\right) s^{2}+\left(-m^{2}+m^{2} \nu-2 m \nu+2 m\right) s=0
$$

The roots of Eq. (3.2) are

$$
s_{1}=0 \quad s_{2}=2-m \quad s_{3}=1-\frac{m}{2}-\mathcal{H} \quad s_{4}=1-\frac{m}{2}+\mathcal{H}
$$

where

$$
\mathcal{H}=\frac{1}{2} \sqrt{m^{2}-4 m \nu+4}
$$

The general solution to Eq. (3.1) is

$$
w(\xi)=C_{1}+C_{2} \xi^{2-m}+C_{3} \xi^{1-\frac{m}{2}-\mathcal{H}}+C_{4} \xi^{1-\frac{m}{2}+\mathcal{H}}
$$

Green's function (solution to the homogeneous Euler equation $L\left(K_{m}(\xi, \alpha)\right)=0$ ) for different values of the power index $m$ may be received from a formula presented in the following form (Jaroszewicz and Zoryj, 2005)

$$
K_{m}(\xi, \alpha)=\frac{A_{m}}{W(\alpha)_{m} p_{0}(\alpha)}
$$

where $p_{0}(\alpha)=1$ is a coefficient placed before the highest order of the derivative of Euler differential equation (3.1) and

$$
\begin{gathered}
A_{m}=\left|\begin{array}{cccc}
1 & \alpha^{2-m} & \alpha^{1-\frac{m}{2}-\mathcal{H}} & \alpha^{1-\frac{m}{2}+\mathcal{H}} \\
0 & \frac{d \alpha^{2-m}}{d \alpha} & \frac{d \alpha^{1-\frac{m}{2}-\mathcal{H}}}{d \alpha} & \frac{d \alpha^{1-\frac{m}{2}+\mathcal{H}}}{d \alpha} \\
0 & \frac{d^{2} \alpha^{2-m}}{d \alpha^{2}} & \frac{d^{2} \alpha^{1-\frac{m}{2}-\mathcal{H}}}{d \alpha^{2}} & \frac{d^{2} \alpha^{1-\frac{m}{2}}+\mathcal{H}}{d \alpha^{2}} \\
1 & \xi^{2-m} & \xi^{1-\frac{m}{2}}-\mathcal{H} & \xi^{1-\frac{m}{2}+\mathcal{H}}
\end{array}\right| \\
W(\alpha)_{m}=\left|\begin{array}{cccc}
1 & \alpha^{2-m} & \alpha^{1-\frac{m}{2}-\mathcal{H}} & \alpha^{1-\frac{m}{2}+\mathcal{H}} \\
0 & \frac{d \alpha^{2-m}}{d \alpha} & \frac{d \alpha^{1-\frac{m}{2}-\mathcal{H}}}{d \alpha} & \frac{d \alpha^{1-\frac{m}{2}+\mathcal{H}}}{d \alpha} \\
0 & \frac{d^{2} \alpha^{2-m}}{d \alpha^{2}} & \frac{d^{2} \alpha^{1-\frac{m}{2}-\mathcal{H}}}{d \alpha^{2}} & \frac{d^{2} \alpha^{1-\frac{m}{2}}+\mathcal{H}}{d \alpha^{2}} \\
0 & \frac{d^{3} \alpha^{2-m}}{d \alpha^{3}} & \frac{d^{3} \alpha^{1-\frac{m}{2}-\mathcal{H}}}{d \alpha^{3}} & \frac{d^{3} \alpha^{1-\frac{m}{2}+\mathcal{H}}}{d \alpha^{3}}
\end{array}\right|
\end{gathered}
$$


The functions $1, \alpha^{2-m}, \alpha^{1-\frac{m}{2}-\mathcal{H}}, \alpha^{1-\frac{m}{2}+\mathcal{H}}$ are linear independent solutions, then the Wronskian must satisfy the condition (Stakgold and Holst, 2011)

$$
W(\alpha)_{m}=-\frac{\mathcal{H}}{8}(-2+m)\left[(-2+m)^{2}-4 \mathcal{H}^{2}\right]^{2} \alpha^{-2(1+m)} \neq 0 \quad \text { for } m \neq 0 \wedge m \neq 2
$$

After calculations, Green's function (GF) has the following form

$$
\begin{aligned}
& K_{m}(\xi, \alpha)=\frac{2 \xi^{-m-\mathcal{H}} \alpha^{1-\mathcal{H}}}{\mathcal{H}(m-2)\left(4-4 m+m^{2}-4 \mathcal{H}^{2}\right)} \\
& \quad \cdot\left[(2-m) \xi^{1+\frac{m}{2}+2 \mathcal{H}} \alpha^{1+\frac{m}{2}}+2 \xi^{m+\mathcal{H}} \mathcal{H} \alpha^{2+\mathcal{H}}-2 \xi^{2+\mathcal{H}} \mathcal{H} \alpha^{m+\mathcal{H}}+(m-2) \xi^{1+\frac{m}{2}} \alpha^{1+\frac{m}{2}+2 \mathcal{H}}\right]
\end{aligned}
$$

and satisfies the conditions

$$
\begin{aligned}
& K_{m}(\alpha, \alpha)=\left.\frac{\partial K_{m}(\xi, \alpha)}{\partial \xi}\right|_{\xi=\alpha}=\left.\frac{\partial^{2} K_{m}(\xi, \alpha)}{\partial \xi^{2}}\right|_{\xi=\alpha}=0 \\
& \left.\frac{\partial^{3} K_{m}(\xi, \alpha)}{\partial \xi^{3}}\right|_{\xi=\alpha}=1
\end{aligned}
$$

according to the properties of influence functions (Kukla, 2009; Stakgold and Holst, 2011).

The function $K_{m}(\xi, \alpha)$ is indeterminate for $m=0$ and $m=2$. After calculation of the imits of the function $K_{m}(\xi, \alpha)$ for $m \rightarrow 0$ and $m \rightarrow 2$, the determinate Green function have the following form

$$
\lim _{m \rightarrow 0} K_{m}(\xi, \alpha)=\frac{\alpha}{4}\left[\alpha^{2}-\xi^{2}+\left(\xi^{2}+\alpha^{2}\right) \ln \frac{\xi}{\alpha}\right]
$$

when Poisson ratio $\nu=0.25$

$$
\lim _{m \rightarrow 2} K_{m}(\xi, \alpha)=\frac{1}{9} \xi^{-\sqrt{\frac{3}{2}}} \alpha^{3}\left[\sqrt{6} \alpha^{-\sqrt{\frac{3}{2}}}\left(\xi^{\sqrt{6}}-\alpha^{\sqrt{6}}\right)-6 \xi^{\sqrt{\frac{3}{2}}}(\ln \xi+\ln \alpha)\right]
$$

and $\nu=0.33$

$$
\lim _{m \rightarrow 2} K_{m}(\xi, \alpha)=\frac{3 \alpha^{3}}{16}\left[\sqrt{3} \xi^{-\frac{2}{\sqrt{3}}} \alpha^{-\frac{2}{\sqrt{3}}}\left(\xi^{\frac{4}{\sqrt{3}}}-\alpha^{\frac{4}{\sqrt{3}}}\right)-4 \ln \xi+4 \ln \alpha\right]
$$

Examples of the formulas of Green's function $K_{m}(\xi \alpha)$ for different values of the power index $m$ $m \in\{-3,-2,-1,0,2,3,4\}$ are presented as in the following:

- for Poisson ratio $\nu=0.25$

$$
\begin{aligned}
& K_{-3}(\xi, \alpha)=\frac{1}{45 \alpha^{2}}\left(4 \xi^{5}-5 \xi^{\frac{9}{2}} \sqrt{\alpha}+5 \sqrt{\xi} \alpha^{\frac{9}{2}}-4 \alpha^{5}\right) \\
& K_{-2}(\xi, \alpha)=\frac{1}{30 \alpha}\left(5 \xi^{4}-5 \alpha^{4}-2 \sqrt{10} \xi^{2+\sqrt{\frac{5}{2}}} \alpha^{2-\sqrt{\frac{5}{2}}}+2 \sqrt{10} \xi^{2-\sqrt{\frac{5}{2}}} \alpha^{2+\sqrt{\frac{5}{2}}}\right) \\
& K_{-1}(\xi, \alpha)=\frac{2}{9}\left(2 \xi^{3}-2 \alpha^{3}-\sqrt{6} \xi^{\frac{3}{2}+\sqrt{\frac{3}{2}}} \alpha^{\frac{3}{2}-\sqrt{\frac{3}{2}}}+\sqrt{6} \xi^{\frac{3}{2}-\sqrt{\frac{3}{2}}} \alpha^{\frac{3}{2}+\sqrt{\frac{3}{2}}}\right) \\
& K_{0}(\xi, \alpha)=\frac{\alpha}{4}\left[\alpha^{2}-\xi^{2}+\left(\xi^{2}+\alpha^{2}\right) \ln \frac{\xi}{\alpha}\right] \\
& K_{2}(\xi, \alpha)=\frac{1}{9} \xi^{-\sqrt{\frac{3}{2}}} \alpha^{3}\left[\sqrt{6} \alpha^{-\sqrt{\frac{3}{2}}}\left(\xi^{\sqrt{6}}-\alpha^{\sqrt{6}}\right)-6 \xi^{\sqrt{\frac{3}{2}}}(\ln \xi+\ln \alpha)\right] \\
& K_{3}(\xi, \alpha)=\frac{2}{45}\left(-10 \alpha^{3}+\frac{10 \alpha^{4}}{\xi}+\sqrt{10} \xi^{-\frac{1}{2}+\sqrt{\frac{5}{2}}} \alpha^{\frac{7}{2}-\sqrt{\frac{5}{2}}}-\sqrt{10} \xi^{-\frac{1}{2}-\sqrt{\frac{5}{2}}} \alpha^{\frac{7}{2}+\sqrt{\frac{5}{2}}}\right) \\
& K_{4}(\xi, \alpha)=\frac{(\xi-\alpha)^{3} \alpha^{2}(\xi+\alpha)}{12 \xi^{3}}
\end{aligned}
$$


— for Poisson ratio $\nu=0.33$

$$
\begin{aligned}
& K_{-3}(\xi, \alpha)=\frac{1}{170}\left(\frac{17 r^{5}}{\alpha^{2}}-17 \alpha^{3}-5 \sqrt{17} \xi^{\frac{1}{2}(5+\sqrt{17})} \alpha^{\frac{1}{2}-\sqrt{\frac{17}{2}}}+5 \sqrt{17} \xi^{\frac{5}{2}-\sqrt{\frac{17}{2}}} \alpha^{\frac{1}{2}(1+\sqrt{17})}\right. \\
& K_{-2}(\xi, \alpha)=\frac{3}{32 \alpha}\left(2 \xi^{4}-2 \alpha^{4}-\sqrt{6} \xi^{2+2 \sqrt{\frac{2}{3}}} \alpha^{2-2 \sqrt{\frac{2}{3}}}+\sqrt{6} \xi^{2-2 \sqrt{\frac{2}{3}}} \alpha^{2+2 \sqrt{\frac{2}{3}}}\right) \\
& K_{-1}(\xi, \alpha)=\frac{1}{38}\left(19 \xi^{3}-19 \alpha^{3}-3 \sqrt{57} \xi^{\frac{1}{6}(9+\sqrt{57})} \alpha^{\frac{1}{6}(9-\sqrt{57})}+3 \sqrt{57} \xi^{\frac{1}{6}(9-\sqrt{57})} \alpha^{\frac{1}{6}(9+\sqrt{57})}\right) \\
& K_{0}(\xi, \alpha)=\frac{\alpha}{4}\left[\alpha^{2}-\xi^{2}+\left(\xi^{2}+\alpha^{2}\right) \ln \frac{\xi}{\alpha}\right] \\
& K_{2}(\xi, \alpha)=\frac{3 \alpha^{3}}{16}\left[\sqrt{3} \xi^{-\frac{2}{\sqrt{3}}} \alpha^{-\frac{2}{\sqrt{3}}}\left(\xi^{\frac{4}{\sqrt{3}}}-\alpha^{\frac{4}{\sqrt{3}}}\right)-4 \ln \xi+4 \ln \alpha\right] \\
& K_{3}(\xi, \alpha)=\frac{(\xi-\alpha)^{3} \alpha^{2}}{6 \xi^{2}} \\
& K_{4}(\xi, \alpha)=\frac{3 \alpha^{3}}{176}\left(\frac{11 \alpha^{2}}{\xi^{2}}+\sqrt{33} \xi^{-1+\sqrt{\frac{11}{3}}} \alpha^{1-\sqrt{\frac{11}{3}}}-\sqrt{33} \xi^{-1-\sqrt{\frac{11}{3}}} \alpha^{1+\sqrt{\frac{11}{3}}}-11\right)
\end{aligned}
$$

\section{Solution of the problem}

The ordinary differential equations with constant or variable coefficients can be transformed to the Volterra or Fredholm integral equations by using e.g. Fubini's method (Pogorzelski, 1958). The solutions to these equations are solutions to the transformed ordinary differential equation. If Green's function (kernel of integral equation) is well known (or determined), the linear independent solutions can be expanded in the Neumann (called Liouville-Neumann) power series rapidly convergent to the eigenvalues (spectrum of integral kernel) based onthe method of successive approximations (Tricomi, 1957; Shestopalov and Smirnov, 2002).

The limited (for $\xi=0$ ) independent solutions of Eq. (3.1) are $w_{1}(\xi)=1$ and $w_{2}(\xi)=\xi^{2-m}$ (or $w_{2}(\xi)=\xi^{1-\frac{m}{2}+\mathcal{H}}$ for $m \geqslant 2$ ). These solutions are expanded in the Neumann power series in the following form

$$
\begin{aligned}
& K_{m}(\xi, \lambda)_{u}=K_{0}(\xi)_{u}+\sum_{i=1}^{\eta} K_{i}(\xi)_{u} \lambda^{2 i} \quad \lambda \in \mathcal{R}^{+} \\
& K_{m}(\xi, \lambda)_{v}=K_{0}(\xi)_{v}+\sum_{i=1}^{\eta} K_{i}(\xi)_{v} \lambda^{2 i}
\end{aligned}
$$

where $K_{i}(\xi)_{u}$ and $K_{i}(\xi)_{v}$ are integral iterated kernels given by

$$
\begin{array}{rlrl}
K_{i}(\xi)_{u} & =\int_{0}^{\xi} K_{m}(\xi, \alpha) \alpha^{-\frac{2}{3} m} K_{i-1}(\alpha)_{u} d \alpha & K_{0}(\alpha)_{u}=\chi_{u} \\
K_{i}(\xi)_{v}=\int_{0}^{\xi} K_{m}(\xi, \alpha) \alpha^{-\frac{2}{3} m} K_{i-1}(\alpha)_{v} d \alpha & K_{0}(\alpha)_{v}=\chi_{v}
\end{array}
$$

and $\eta$ is the degree of approximations. $\chi_{u}$ and $\chi_{v}$ are limited independent solutions to Eq. (3.1) for $\xi=0 . \chi_{u}=1$ for all values of the parameter $m$. Values of $\chi_{v}$ depend on the power index $m$ and the Poisson ratio $\nu$ (for $m \geqslant 2$ ). They are shown in Table 1 . 
Table 1. Values of $\chi_{v}$ for some considered values of the power index $m$

\begin{tabular}{|c|c|c|c|c|c|c|c|}
\hline$m$ & -3 & -2 & -1 & 0 & 2 & 3 & 4 \\
\hline$\chi_{v}$ & $\alpha^{5}$ & $\alpha^{4}$ & $\alpha^{3}$ & $\alpha^{2}$ & $\alpha^{2 \sqrt{3} / 3}$ & $\alpha$ & $\alpha^{-1+\sqrt{11 / 3}}$ \\
\hline
\end{tabular}

The characteristic equations $\Delta_{m}=0$ for different boundary conditions and different values of the parameter $m$ are obtained from well known characteristic determinants given by:

- clamped

$$
\Delta_{m}(\lambda) \equiv\left|\begin{array}{cc}
K_{m}(\xi, \lambda)_{u} & K_{m}(\xi, \lambda)_{v} \\
\frac{\partial K_{m}(\xi, \lambda)_{u}}{\partial \xi} & \frac{\partial K_{m}(\xi, \lambda)_{v}}{\partial \xi}
\end{array}\right|_{\xi=1}
$$

— simply supported

$$
\Delta_{m}(\lambda) \equiv\left|\begin{array}{cc}
K_{m}(\xi, \lambda)_{u} & K_{m}(\xi, \lambda)_{v} \\
M\left[K_{m}(\xi, \lambda)_{u}\right] & M\left[K_{m}(\xi, \lambda)_{v}\right]
\end{array}\right|_{\xi=1}
$$

- free

$$
\Delta_{m}(\lambda) \equiv\left|\begin{array}{ll}
M\left[K_{m}(\xi, \lambda)_{u}\right] & M\left[K_{m}(\xi, \lambda)_{v}\right] \\
V\left[K_{m}(\xi, \lambda)_{u}\right] & V\left[K_{m}(\xi, \lambda)_{v}\right]
\end{array}\right|_{\xi=1}
$$

— sliding supports

$$
\Delta_{m}(\lambda) \equiv\left|\begin{array}{cc}
\frac{\partial K_{m}(\xi, \lambda)_{u}}{\partial \xi} & \frac{\partial K_{m}(\xi, \lambda)_{v}}{\partial \xi} \\
V\left[K_{m}(\xi, \lambda)_{u}\right] & V\left[K_{m}(\xi, \lambda)_{v}\right]
\end{array}\right|_{\xi=1}
$$

- elastic supports

$$
\Delta_{m}(\lambda) \equiv\left|\begin{array}{ll}
\Phi\left[K_{m}(\xi, \lambda)_{u}\right] & \Phi\left[K_{m}(\xi, \lambda)_{v}\right] \\
\Psi\left[K_{m}(\xi, \lambda)_{u}\right] & \Psi\left[K_{m}(\xi, \lambda)_{v}\right]
\end{array}\right|_{\xi=1}
$$

For all boundary conditions, the formula of $\Delta_{m}$ has the following form

$$
\Delta_{m}=a_{0}+\sum_{i=1}^{\eta}(-1)^{i} a_{i} \lambda^{2 i}
$$

where $a_{0}, a_{1}, \ldots, a_{\eta}$ are coefficients of characteristic equations depending on the boundary conditions and the parameter $m$.

\section{Results and discussion}

The numerical results for dimensionless frequencies of the uniform and non-uniform circular plates with different boundary conditions are presented in Tables 2-5. The Neumann power series (Eq. (4.1)) expanded only for $\eta=15$ allows one to obtained six lower exact eigenvalues for all considered cases. The numerical dimensionless frequencies of the uniform circular plates are presented in Table 2 with comparison to the results by Duan et al. (2014), Leissa (1969), Wu and Liu (2002) and Yalcin et al. (2009). The numerical results for uniform circular plates with elastic supports are shown in Table 3 with comparison to the results by Wu and Liu (2002). 
Table 2. The first six lower dimensionless frequencies $\lambda=\omega R^{2} \sqrt{\rho h_{R} / D_{R}}$ of the uniform circular plates

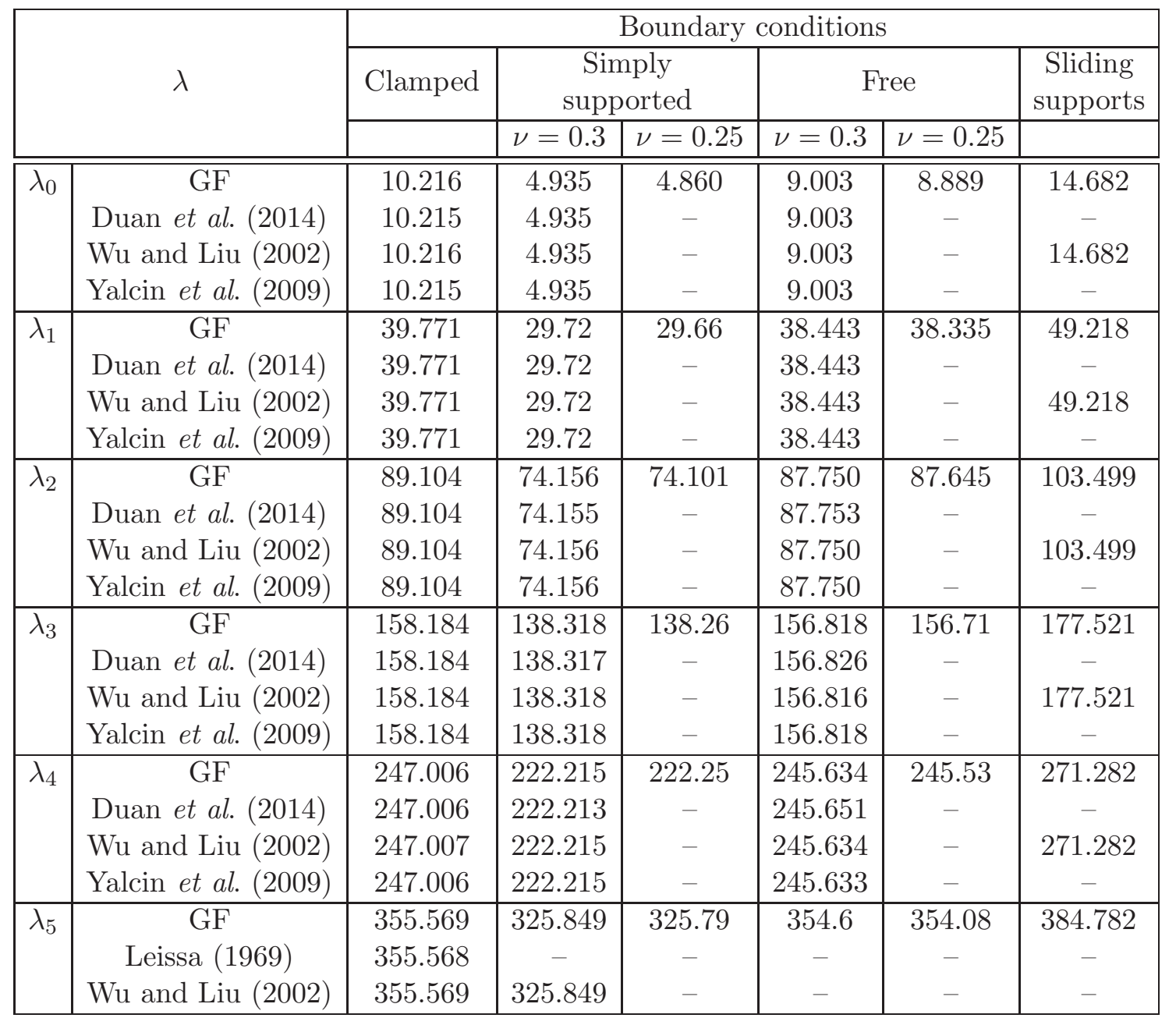

GF - Green's function

Table 3. The first six lower dimensionless frequencies $\lambda=\omega R^{2} \sqrt{\rho h_{R} / D_{R}}$ of the uniform circular plates with elastic supports, Poisson ratio $\nu=0.3$

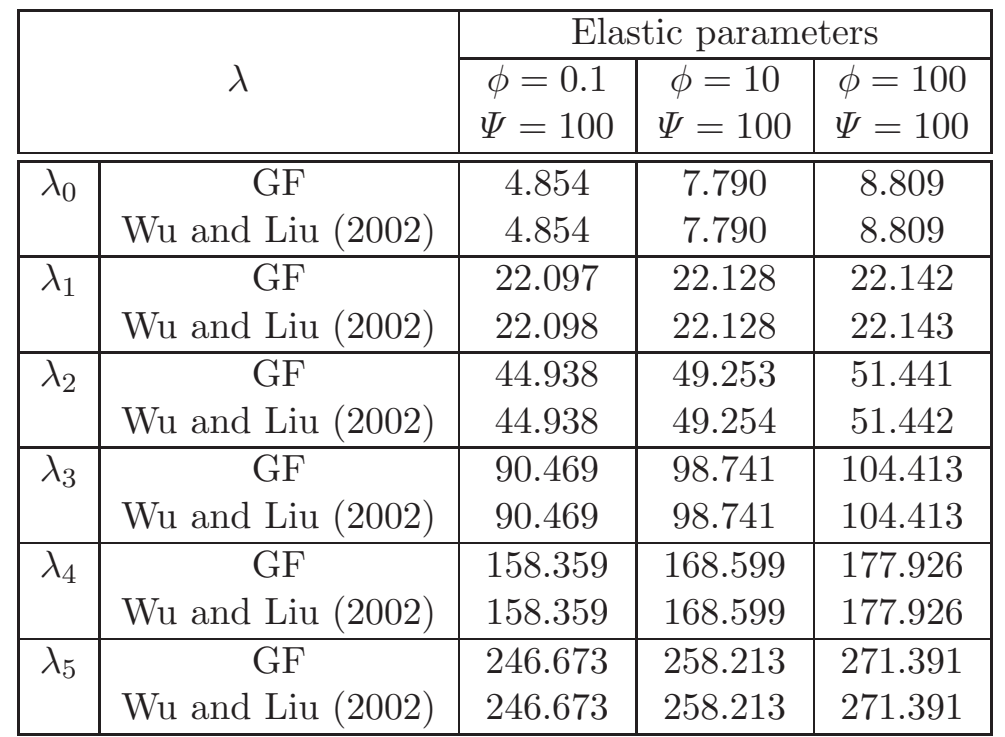


Table 4. The first six lower dimensionless frequencies $\lambda=\omega R^{m / 3} \sqrt{\rho h_{R} / D_{R}}$ of the non-uniform circular plates

\begin{tabular}{|c|c|c|c|c|c|c|c|c|c|}
\hline \multirow{3}{*}{$m$} & \multirow{3}{*}{$\lambda$} & \multicolumn{8}{|c|}{ Boundary conditions } \\
\hline & & \multicolumn{2}{|c|}{ Clamped } & \multicolumn{2}{|c|}{$\begin{array}{c}\text { Simply } \\
\text { supported }\end{array}$} & \multicolumn{2}{|c|}{ Free } & \multicolumn{2}{|c|}{$\begin{array}{c}\text { Sliding } \\
\text { supports }\end{array}$} \\
\hline & & $\nu=0.33$ & $\nu=0.25$ & $\nu=0.33$ & $\nu=0.25$ & $\nu=0.33$ & $\nu=0.25$ & $\nu=0.33$ & $\nu=0.25$ \\
\hline \multirow{6}{*}{-3} & $\overline{\lambda_{0}}$ & 16.902 & $\overline{17.209}$ & 10.851 & 10.981 & 25.643 & 25.501 & 36.543 & 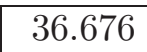 \\
\hline & $\lambda_{1}$ & 86.044 & 86.188 & 67.382 & 67.403 & 90.847 & 90.722 & 114.63 & 114.75 \\
\hline & $\lambda_{2}$ & 197.11 & 197.25 & 167.37 & 167.38 & 210.79 & 201.67 & 236.94 & 237.07 \\
\hline & $\lambda_{3}$ & 352.64 & 352.78 & 311.75 & 311.76 & 357.14 & 357.01 & 403.59 & 403.71 \\
\hline & $\lambda_{4}$ & 552.55 & 552.69 & 500.53 & 500.54 & 556.94 & 556.82 & 614.61 & 614.74 \\
\hline & $\lambda_{5}$ & 796.86 & 796.99 & 733.71 & 733.72 & 801.18 & 801.05 & 870.03 & 870.16 \\
\hline \multirow{6}{*}{-2} & $\lambda_{0}$ & 15.147 & 15.331 & 9.280 & 9.314 & 19.555 & 19.398 & 28.537 & 28.625 \\
\hline & $\lambda_{1}$ & 68.932 & 69.027 & 53.458 & 53.440 & 71.203 & 71.062 & 90.109 & 90.192 \\
\hline & $\lambda_{2}$ & 156.66 & 156.75 & 132.43 & 132.41 & 158.85 & 158.71 & 186.69 & 186.77 \\
\hline & $\lambda_{3}$ & 279.52 & 279.61 & 246.49 & 246.47 & 281.60 & 281.46 & 318.33 & 318.41 \\
\hline & $\lambda_{4}$ & 437.46 & 437.54 & 395.64 & 395.61 & 439.47 & 439.33 & 485.04 & 485.13 \\
\hline & $\lambda_{5}$ & 630.48 & 630.56 & 579.87 & 579.85 & 632.45 & 632.31 & 686.84 & 686.92 \\
\hline \multirow{6}{*}{-1} & $\lambda_{0}$ & 12.868 & 12.951 & 7.302 & 7.256 & 14.041 & 13.868 & 21.254 & 21.297 \\
\hline & $\lambda_{1}$ & 53.504 & 53.551 & 40.917 & 40.860 & 53.762 & 53.604 & 68.307 & 68.349 \\
\hline & $\lambda_{2}$ & 120.65 & 120.70 & 101.37 & 101.31 & 120.86 & 120.70 & 142.21 & 142.25 \\
\hline & $\lambda_{3}$ & 214.70 & 214.74 & 188.69 & 188.63 & 214.85 & 214.69 & 242.97 & 243.01 \\
\hline & $\lambda_{4}$ & 335.61 & 335.65 & 302.88 & 302.82 & 335.72 & 335.57 & 370.60 & 370.64 \\
\hline & $\lambda_{5}$ & 483.38 & 483.42 & 443.93 & 443.87 & 483.48 & 483.32 & 525.09 & 525.13 \\
\hline \multirow{6}{*}{2} & $\lambda_{0}$ & 8.894 & 9.111 & 3.297 & 3.334 & 5.302 & 5.412 & 8.876 & 9.193 \\
\hline & $\lambda_{1}$ & 25.837 & 26.306 & 19.076 & 19.410 & 22.951 & 23.296 & 28.472 & 29.011 \\
\hline & $\lambda_{2}$ & 51.575 & 52.278 & 42.759 & 43.337 & 48.776 & 49.363 & 56.510 & 57.279 \\
\hline & $\lambda_{3}$ & 86.082 & 87.017 & 75.135 & 75.949 & 83.323 & 84.144 & 93.262 & 94.260 \\
\hline & $\lambda_{4}$ & 129.36 & 130.52 & 116.25 & 117.30 & 126.62 & 127.67 & 138.76 & 139.99 \\
\hline & $\lambda_{5}$ & 181.41 & 182.81 & 166.13 & 167.41 & 178.68 & 179.97 & 193.03 & 194.49 \\
\hline \multirow{13}{*}{3} & $\lambda_{0}$ & 8.719 & 8.965 & 3.002 & 3.073 & 4.686 & 4.843 & 8.787 & 7.170 \\
\hline & {$[1]$} & 8.720 & - & - & - & - & - & - & - \\
\hline & {$[7]$} & 8.708 & - & - & - & - & - & - & - \\
\hline & {$[16]$} & 8.719 & 8.965 & - & - & - & - & - & - \\
\hline & $\lambda_{1}$ & 21.145 & 21.609 & 15.761 & 16.110 & 18.152 & 18.520 & 21.638 & 22.170 \\
\hline & [1] & 21.15 & - & - & - & - & - & - & - \\
\hline & [16] & 21.145 & 21.609 & - & - & - & - & - & - \\
\hline & $\lambda_{2}$ & 38.453 & 39.122 & 32.031 & 32.595 & 35.607 & 36.187 & 40.402 & 41.133 \\
\hline & {$[1]$} & 38.45 & - & - & - & - & - & - & - \\
\hline & [16] & 38.453 & 39.122 & - & - & - & - & - & - \\
\hline & $\lambda_{3}$ & 60.680 & 61.551 & 53.108 & 53.879 & 57.892 & 58.677 & 63.964 & 64.894 \\
\hline & $\lambda_{4}$ & 87.834 & 88.910 & 79.076 & 80.052 & 85.077 & 86.066 & 92.411 & 93.577 \\
\hline & $\lambda_{5}$ & 119.91 & 121.19 & 109.95 & 111.03 & 117.18 & 118.31 & 125.78 & 126.78 \\
\hline \multirow{6}{*}{4} & $\lambda_{0}$ & 8.458 & 8.705 & 2.877 & 2.965 & 4.395 & 4.569 & 3.644 & 4.273 \\
\hline & $\lambda_{1}$ & 16.735 & 17.137 & 12.781 & 13.096 & 14.093 & 14.427 & 15.778 & 16.236 \\
\hline & $\lambda_{2}$ & 27.094 & 27.643 & 22.827 & 23.299 & 24.645 & 25.131 & 27.042 & 27.639 \\
\hline & $\lambda_{3}$ & 39.611 & 40.303 & 34.898 & 35.418 & 37.240 & 37.872 & 40.255 & 41.007 \\
\hline & $\lambda_{4}$ & 54.305 & 55.139 & 49.123 & 49.866 & 51.975 & 52.751 & 55.925 & 56.484 \\
\hline & $\lambda_{5}$ & 70.806 & 71.542 & 65.087 & 66.243 & 68.884 & 69.791 & 70.875 & 74.118 \\
\hline
\end{tabular}

[1] - Conway (1957), [7] - Jaroszewicz and Zoryj (2006), [16] - Wang (1997) 
Table 5. The first six lower dimensionless frequencies $\lambda=\omega R^{m / 3} \sqrt{\rho h_{R} / D_{R}}$ of the non-uniform circular plates with elastic supports

\begin{tabular}{|c|c|c|c|c|c|c|c|}
\hline \multirow{3}{*}{$m$} & \multirow{3}{*}{$\lambda$} & \multicolumn{6}{|c|}{ Elastic parameters } \\
\hline & & \multicolumn{2}{|c|}{$\phi=0.1, \Psi=10$} & \multicolumn{2}{|c|}{$\phi=100, \Psi=10$} & \multicolumn{2}{|c|}{$\phi=10, \Psi=10$} \\
\hline & & $\nu=0.33$ & $\nu=0.25$ & $\nu=0.33$ & $\nu=0.25$ & $\nu=0.33$ & $\nu=0.25$ \\
\hline \multirow{6}{*}{-3} & $\lambda_{0}$ & 3.595 & 3.670 & 2.965 & 3.011 & 0.317 & 0.322 \\
\hline & $\lambda_{1}$ & 27.042 & 26.920 & 36.525 & 36.650 & 33.285 & 33.379 \\
\hline & $\lambda_{2}$ & 91.487 & 91.369 & 113.22 & 113.34 & 104.64 & 104.72 \\
\hline & $\lambda_{3}$ & 202.25 & 202.13 & 233.81 & 233.93 & 218.85 & 218.90 \\
\hline & $\lambda_{4}$ & 357.53 & 357.41 & 398.25 & 398.37 & 376.43 & 376.46 \\
\hline & $\lambda_{5}$ & 557.30 & 557.18 & 606.59 & 606.71 & 577.83 & 577.84 \\
\hline \multirow{6}{*}{-2} & $\lambda_{0}$ & 3.818 & 3.873 & 3.344 & 3.377 & 0.354 & 0.357 \\
\hline & $\lambda_{1}$ & 21.143 & 21.012 & 28.709 & 28.790 & 26.104 & 26.164 \\
\hline & $\lambda_{2}$ & 71.857 & 71.723 & 89.165 & 89.246 & 82.728 & 82.772 \\
\hline & $\lambda_{3}$ & 159.29 & 159.16 & 184.49 & 184.58 & 173.21 & 173.23 \\
\hline & $\lambda_{4}$ & 281.972 & 281.83 & 314.55 & 314.63 & 297.95 & 297.95 \\
\hline & $\lambda_{5}$ & 439.81 & 439.67 & 479.34 & 479.42 & 457.28 & 457.27 \\
\hline \multirow{6}{*}{-1} & $\lambda_{0}$ & 3.860 & 3.886 & 3.749 & 3.769 & 0.394 & 0.396 \\
\hline & $\lambda_{1}$ & 15.947 & 15.818 & 21.624 & 21.662 & 19.520 & 19.546 \\
\hline & $\lambda_{2}$ & 54.447 & 54.297 & 67.746 & 67.787 & 63.101 & 63.114 \\
\hline & $\lambda_{3}$ & 121.29 & 121.14 & 140.76 & 140.80 & 132.60 & 132.60 \\
\hline & $\lambda_{4}$ & 215.20 & 215.04 & 240.43 & 240.47 & 228.33 & 228.31 \\
\hline & $\lambda_{5}$ & 336.06 & 335.88 & 366.76 & 366.77 & 350.51 & 350.48 \\
\hline \multirow{6}{*}{2} & $\lambda_{0}$ & 3.140 & 3.168 & 7.330 & 7.148 & 0.729 & 0.723 \\
\hline & $\lambda_{1}$ & 9.061 & 9.195 & 8.855 & 9.317 & 8.250 & 8.546 \\
\hline & $\lambda_{2}$ & 23.752 & 24.096 & 28.495 & 29.027 & 26.982 & 27.482 \\
\hline & $\lambda_{3}$ & 49.197 & 49.784 & 56.260 & 57.022 & 53.862 & 54.581 \\
\hline & $\lambda_{4}$ & 83.620 & 84.442 & 92.740 & 93.731 & 89.265 & 90.204 \\
\hline & $\lambda_{5}$ & 126.86 & 127.918 & 137.94 & 139.16 & 133.27 & 134.43 \\
\hline \multirow{6}{*}{3} & $\lambda_{0}$ & 3.100 & 3.156 & 21.623 & 22.157 & 1.050 & 1.023 \\
\hline & $\lambda_{1}$ & 8.804 & 8.954 & 40.302 & 41.028 & 6.374 & 6.733 \\
\hline & $\lambda_{2}$ & 18.947 & 19.309 & 63.727 & 64.651 & 20.771 & 21.271 \\
\hline & $\lambda_{3}$ & 36.010 & 36.589 & 92.023 & 93.149 & 38.923 & 39.615 \\
\hline & $\lambda_{4}$ & 58.170 & 58.955 & 125.22 & 126.24 & 61.783 & 62.668 \\
\hline & $\lambda_{5}$ & 85.295 & 86.304 & 153.08 & 155.33 & 89.454 & 90.552 \\
\hline \multirow{6}{*}{4} & $\lambda_{0}$ & 3.166 & 3.239 & 15.354 & 15.859 & 2.642 & 1.999 \\
\hline & $\lambda_{1}$ & 8.651 & 8.803 & 26.962 & 27.562 & 15.401 & 15.832 \\
\hline & $\lambda_{2}$ & 14.881 & 15.204 & 40.184 & 40.919 & 26.395 & 26.966 \\
\hline & $\lambda_{3}$ & 25.028 & 25.512 & 55.473 & 56.347 & 39.575 & 40.040 \\
\hline & $\lambda_{4}$ & 37.810 & 38.129 & 72.903 & 73.921 & 48.253 & 55.200 \\
\hline & $\lambda_{5}$ & 45.062 & 52.949 & 92.495 & 93.111 & 59.544 & 72.493 \\
\hline
\end{tabular}

The dimensionless frequencies of the non-uniform circular plates with different boundary conditions are presented in Table 4 with comparison to the results by Conway (1957), Jaroszewicz and Zoryj (2006), Wang (1997). The numerical results for the non-uniform circular plates with elastic supports are shown in Table 5.

The dimensionless frequencies of the non-uniform circular plate (Table 4) decrease when values of the power index increase. However, the absolute values of frequencies $\omega$ increase if the power index increases, which is according to physical properties of this kind of plates with 
variable thickness (Wu and Liu, 2002). Additionally, the dimensionless frequencies depend on functions describing the distribution of plate parameters such as thickness or rigidity. The dimensionless frequencies and absolute values $\omega$ for the uniform and the non-uniform circular plates with elastic constraints (Table 3 and 5) depend on combination of values of the elastic parameters.

\section{Conclusions}

In this paper, Green's functions have been employed to solve the problem of natural vibration of uniform and non-uniform circular thin plates with different boundary conditions. The universal Green function for different power indices $m$ and different Poisson ratios is defined. The limited solutions to the Euler equation expanded in the Neumann power series allow one to obtain characteristic equations of circular plates rapidly convergent to the exact eigenvalues. The characteristic equations have been obtained for different values of the parameter $m$, different values of Poisson's ratio and different boundary conditions. The considered values of Poisson's ratio have not large influence on the dimensionless eigenvalues, but the numerical results of the investigation can be used to validate the accuracy of other numerical methods as benchmark values. The obtained results are in good agreement with the results obtained by other methods presented in the literature. The calculations have been carried out with the help of Mathematica v10, which is a symbolic calculation software.

\section{References}

1. Conway H.D., 1957, An analogy between the flexural vibrations of a cone and a disc of linearly varying thickness, Journal of Applied Mathematics and Mechanics, 37, 9, 406-407

2. Conway H.D., 1958, Some special solutions for the flexural vibrations of discs of varying thickness, Ingenieur-Archiv, 26, 6, 408-410

3. Duan G., Wang X., Jin Ch., 2014, Free vibration analysis of circular thin plates with stepped thickness by the DSC element method, Thin-Walled Structures, 85, 25-33

4. Gupta U.S., Lal R., Sharma S., 2006, Vibration analysis of non-homogeneous circular plate of nonlinear thickness variation by differential quadrature method, Journal of Sound and Vibration, 298, 892-906

5. Jain R.K., Prasad C., Soni S.R., 1972, Axisymmetric vibrations of circular plates of linearly varying thickness, $Z A M P, \mathbf{2 3}, 941-947$

6. Jaroszewicz J., Zoryj L., 2005, Methods of Free Axisymmetric Vibration Analysis of Circular Plates Using by Influence Functions, Bialystok University of Technology, Poland

7. Jaroszewicz J., ZoryJ L., 2006, The method of partial discretization in free vibration problems of circular plates with variable distribution of parameters, International Applied Mechanics, 42, 364-373

8. Kukla S., 2009, Green's Functions and their Properties, Czestochowa University of Technology, Poland

9. LeIsSA A.W., 1969, Vibration of Plates, Washington

10. Pogorzelski W., 1958, Integral Equations and their Applications, Vol. 1, PWN Warszawa

11. Shestopalov Y.V, Smirnov Y.G., 2002, Integral Equations, Karlstad

12. Stakgold I., Holst M., 2011, Green's Functions and Boundary Value Problem, Edition 3rd, Wiley 
13. Taher H.R., Omidi M., Zadpoor A.A., Nikooyan A.A., 2006, Free vibration of circular and annular plates with variable thickness and different combinations of boundary conditions, Journal of Sound and Vibration, 296, 1084-1092

14. Timoshenko S., Woinowsky-Krieger S., 1959, Theory of Plates and Shells, McGraw-Hill, New York

15. Tricomi F.G., 1957, Integral Equations, New York

16. WANG J., 1997, General power series solution of the vibration of classical circular plates with variable thickness, Journal of Sound and Vibration, 202, 593-599

17. Wu T.Y., LiU G.R., 2001, Free vibration analysis of circular plates with variable thickness by the generalized differential quadrature rule, International Journal of Solids and Structures, 38, 7967-7980

18. Wu T.Y., LIU G.R., 2002, Free vibration analysis of circular plates using generalized differential quadrature rule, Computer Methods in Applied Mechanics and Engineering, 191, 5365-5380

19. Yalcin H.S., Arikoglu A., Ozkol I., 2009, Free vibration analysis of circular plates by differential transformation method, Applied Mathematics and Computation, 212, 2, 377-386

20. YAng J.S., 1993, The vibration of circular plate with variable thickness, Journal of Sound and Vibration, 165, 178-185

21. Zhou Z.H., Wong K.W., Xu X.S., Leung A.Y.T., 2011, Natural vibration of circular and annular plates by Hamiltonian approach, Journal of Sound and Vibration, 330, 5, 1005-1017

Manuscript received October 2, 2014; accepted for print May 4, 2015 